\title{
DETECTION OF A NEW NONTRIVIAL FAMILY IN THE STABLE HOMOTOPY OF SPHERES $\pi_{*} S$
}

\author{
LIU XIUGUI AND JIN YINGLONG
}

\begin{abstract}
To determine the stable homotopy groups of spheres is one of the central problems in homotopy theory. Let $A$ be the $\bmod p$ Steenrod algebra and $S$ the sphere spectrum localized at an odd prime $p$. In this article, it is proved that for $p \geqslant 7, n \geqslant 4$ and $3 \leqslant s<p-2$, the product $b_{0} h_{1} h_{n} \tilde{\gamma}_{s} \in E x t_{A}^{s+4, *}\left(\mathbb{Z}_{p}, \mathbb{Z}_{p}\right)$ is a permanent cycle in the Adams spectral sequence and converges to a nontrivial element of order $p$ in the stable homotopy groups of spheres $\pi_{p^{n} q+s p^{2} q+(s+1) p q+(s-2) q-7} S$, where $q=2(p-1)$.
\end{abstract}

\section{Introduction and statement of the main theorem}

The $i$-th homotopy group $\pi_{i}(X)$ of a topological space $X$ is considered as the set of homotopy classes of the mappings from $i$-sphere $S^{i}$ into $X$ preserving base points. One of the main problems in homotopy theory is to determine the homotopy groups $\pi_{i}\left(S^{n}\right)$ of spheres, since this is the first fundamental difficulty in the computations of the homotopy groups of polyhedra and topological spaces.

Throughout this article, we let $A$ denote the $\bmod p$ Steenrod algebra and $S$ denote the sphere spectrum localized at a prime $p \geqslant 7$. To determine the stable homotopy groups of spheres $\pi_{*} S$ is one of the central problems in homotopy theory. One of the main tools to reach it is the Adams spectral sequence: $E_{2}^{s, t}=\operatorname{Ext} t_{A}^{s, t}\left(\mathbb{Z}_{p}, \mathbb{Z}_{p}\right) \Rightarrow \pi_{t-s} S$, where the $E_{2}^{s, t}$-term is the cohomology of $A$.

If a family of homotopy generators $x_{i}$ in $E_{2}^{s, *}$ converges nontrivially in the Adams spectral sequence, then we get a family of homotopy elements $f_{i}$ in $\pi_{*} S$ and we say that $f_{i}$ is represented by $x_{i} \in E_{2}^{s, *}$ and has filtration $s$ in the Adams spectral sequence. So far, not so many families of homotopy elements in $\pi_{*} S$ have been detected. Recently, Lin Jinkun got a series of results and detected some new families in $\pi_{*} S$.

In this article, we always fix $q=2(p-1)$.

Received November 23, 2005.

2000 Mathematics Subject Classification. 55Q45.

Key words and phrases. Stable homotopy groups of spheres, Adams spectral sequence, May spectral sequence.

The research is partially supported by the National Natural Science Foundation of China (Nos. 10501045, 10771105) and the Fund of the Personnel Division of Nankai University(No. J02017). 
Theorem 1.1.([1]) Let $p \geqslant 7, n \geqslant 4$, then the product $b_{n-1} g_{0} \tilde{\gamma}_{3} \neq 0 \in \operatorname{Ext}_{A}^{7, *}\left(\mathbb{Z}_{p}, \mathbb{Z}_{p}\right)$ and it converges in the Adams spectral sequence to a nontrivial element in

$\pi_{p^{n} q+3\left(p^{2}+p+1\right) q-7} S$ of order $p$.

Theorem 1.2.([2]) Let $p \geqslant 7, n \geqslant 4$, then $h_{n} g_{0} \tilde{\gamma}_{3} \neq 0 \in E x t_{A}^{6, *}\left(\mathbb{Z}_{p}, \mathbb{Z}_{p}\right)$ and it converges in the Adams spectral sequence to a nontrivial element in $\pi_{p^{n} q+3\left(p^{2}+p+1\right) q-6}$ S of order $p$.

Theorem 1.3.([3]) Let $p \geqslant 5, n \geqslant 3$, then,

(1) $i_{*}\left(h_{1} h_{n}\right) \neq 0 \in \operatorname{Ext}_{A}^{2, p^{n} q+p q}\left(H^{*} M, \mathbb{Z}_{p}\right)$ is a permanent cycle in the Adams spectral sequence and converges to a nontrivial element $\xi_{n} \in \pi_{p^{n} q+p q-2} M$.

(2) For $\xi_{n} \in \pi_{p^{n} q+p q-2} M$ obtained in (1), $j \xi_{n} \in \pi_{p^{n} q+p q-3} S$ is a nontrivial element of order $p$ which is represented up to nonzero scalar by $\left(b_{0} h_{n}+h_{1} b_{n-1}\right) \in E x t_{A}^{3, p^{n} q+p q}\left(\mathbb{Z}_{p}, \mathbb{Z}_{p}\right)$ in the Adams spectral sequence.

we use Theorem 1.3 to detect a new family in $\pi_{*} S$. Our result can be stated as follows.

Theorem 1.4. Let $p \geqslant 7, n \geqslant 4$, then the product $b_{0} h_{1} h_{n} \tilde{\gamma}_{s+3} \neq 0 \in E x t_{A}^{s+7, *}\left(\mathbb{Z}_{p}, \mathbb{Z}_{p}\right)$ is a permanent cycle in the Adams spectral sequence and converges to a nontrivial element of order $p$ in $\pi_{p^{n} q+(s+3) p^{2} q+(s+4) p q+(s+1) q-7} S$, where $0 \leqslant s<p-5$.

Remark. The element $b_{0} h_{1} h_{n} \tilde{\gamma}_{s}$ obtained in Theorem 1.4 is an indecomposable element in the stable homotopy groups of spheres $\pi_{*} S$, i.e., it is not a composition of two elements of lower filtration in $\pi_{*} S$, because $h_{n}(n>0)$ is known to die in the Adams spectral sequence.

The article is arranged as follows: after recalling some knowledge on the May spectral sequence in Section 2, we will make use of the May spectral sequence and the Adams spectral sequence to prove Theorem 1.4 in Section 3.

\section{Recollections on the May spectral sequence}

From [4], $E x t_{A}^{1, *}\left(\mathbb{Z}_{p}, \mathbb{Z}_{p}\right)$ has $\mathbb{Z}_{p}$-bases consisting of $a_{0} \in \operatorname{Ext}_{A}^{1,1}\left(\mathbb{Z}_{p}, \mathbb{Z}_{p}\right), h_{i} \in \operatorname{Ext}_{A}^{1, p^{i} q}$ $\left(\mathbb{Z}_{p}, \mathbb{Z}_{p}\right)$ for all $i \geqslant 0$ and $E x t_{A}^{2, *}\left(\mathbb{Z}_{p}, \mathbb{Z}_{p}\right)$ has $\mathbb{Z}_{p}$-bases consisting of $\alpha_{2}, a_{0}^{2}, a_{0} h_{i}(i>0), g_{i}(i \geqslant$ $0), k_{i}(i \geqslant 0), b_{i}(i \geqslant 0)$, and $h_{i} h_{j}(j \geqslant i+2, i \geqslant 0)$ whose internal degrees are $2 q+1,2, p^{i} q+$ $1, p^{i+1} q+2 p^{i} q, 2 p^{i+1} q+p^{i} q, p^{i+1} q$ and $p^{i} q+p^{j} q$ respectively.

From [5], there is a May spectral sequence $\left\{E_{r}^{s, t, *}, d_{r}\right\}$ which converges to $\operatorname{Ext}_{A}^{s, t}\left(\mathbb{Z}_{p}, \mathbb{Z}_{p}\right)$ with $E_{1}$-term

$$
E_{1}^{*, *, *}=E\left(h_{m, i} \mid m>0, i \geqslant 0\right) \bigotimes P\left(b_{m, i} \mid m>0, i \geqslant 0\right) \bigotimes P\left(a_{n} \mid n \geqslant 0\right),
$$

where $E$ is the exterior algebra, $P$ is the polynomial algebra, $h_{m, i} \in E_{1}^{1,2\left(p^{m}-1\right) p^{i}, 2 m-1}, b_{m, i} \in$ $E_{1}^{2,2\left(p^{m}-1\right) p^{i+1}, p(2 m-1)}, a_{n} \in E_{1}^{1,2 p^{n}-1,2 n+1}$. One has $d_{r}: E_{r}^{s, t, u} \rightarrow E_{r}^{s+1, t, u-r}$ and if $x \in E_{r}^{s, t, *}$ and $y \in E_{r}^{s^{\prime}, t^{\prime}, *}$, then $d_{r}(x \cdot y)=d_{r}(x) \cdot y+(-1)^{s} x \cdot d_{r}(y)$. For $x, y=h_{m, i}, b_{m, i}$ or $a_{n}$, we have $x \cdot y=$ $(-1)^{s s^{\prime}+t t^{\prime}} y \cdot x$. 
The first May differential $d_{1}$ is given by

$$
d_{1}\left(h_{i, j}\right)=\sum_{0<k<i} h_{i-k, k+j} h_{k, j}, d_{1}\left(a_{i}\right)=\sum_{0 \leqslant k<i} h_{i-k, k} a_{k}, d_{1}\left(b_{i, j}\right)=0 .
$$

For each element $x \in E_{1}^{s, t, *}$, we define $\operatorname{dim} x=s, \operatorname{deg} x=t$. Then we have:

$$
\left\{\begin{array}{l}
\operatorname{dim} h_{i, j}=\operatorname{dim} a_{i}=1, \quad \operatorname{dim} b_{i, j}=2, \quad \operatorname{deg} a_{0}=1 \\
\operatorname{deg} h_{i, j}=2\left(p^{i}-1\right) p^{j}=2(p-1)\left(p^{i+j-1}+\cdots+p^{j}\right) \\
\operatorname{deg} b_{i, j}=2\left(p^{i}-1\right) p^{j+1}=2(p-1)\left(p^{i+j}+\cdots+p^{j+1}\right) \\
\operatorname{deg} a_{i}=2 p^{i}-1=2(p-1)\left(p^{i-1}+\cdots+1\right)+1
\end{array}\right.
$$

where $i \geqslant 1, j \geqslant 0$.

Proposition 2.1.([6, proposition 1.1]) Let $t=q\left(c_{n} p^{n}+c_{n-1} p^{n-1}+\cdots+c_{1} p+c_{0}\right)+e$ be a positive integer with $0 \leqslant c_{i}<p(0 \leqslant i \leqslant n), 0 \leqslant e<q$, and $s$ a positive integer with $0<s<p$. If for some $j(0 \leqslant j \leqslant n), s<c_{j}$, then we have $E_{1}^{s, t, *}=0$ in the May spectral sequence.

\section{The convergence of the products $b_{0} h_{1} h_{n} \tilde{\gamma}_{s+3}$}

Let $M$ be the Moore spectrum modulo a prime $p \geqslant 5$ given by the cofibration $S \stackrel{p}{\rightarrow} S \stackrel{i}{\rightarrow} M \stackrel{j}{\rightarrow}$ $\Sigma S$. Let $\alpha: \Sigma^{q} M \rightarrow M$ be the Adams map and $K$ be its cofibre given by the cofibration $\Sigma^{q} M \stackrel{\alpha}{\rightarrow}$ $M \stackrel{i^{\prime}}{\rightarrow} K \stackrel{j^{\prime}}{\rightarrow} \Sigma^{q+1} M$. This spectrum which we briefly write as $K$ is known to be the TodaSmith spectrum $V(1)$. Let $V(2)$ be the cofibre of $\beta: \Sigma^{(p+1) q} K \rightarrow K$ given by the cofibration $\Sigma^{(p+1) q} K \stackrel{\beta}{\rightarrow} K \stackrel{\bar{i}}{\rightarrow} V(2) \stackrel{\bar{j}}{\rightarrow} \Sigma^{(p+1) q+1} K$. Let $\gamma: \Sigma^{q\left(p^{2}+p+1\right)} V(2) \rightarrow V(2)$ be the $v_{3}$-map. AS we know, in the Adams spectral sequence, for $p \geqslant 7$ the $\gamma$-element $\gamma_{t}=j j^{\prime} \bar{j} \gamma^{t} \bar{i} i^{\prime} i$ is a nontrivial element of order $p$ in $\pi_{t q\left(p^{2}+p+1\right)-q(p+2)-3} S$ (see [7, Theorem 2.12]).

Proposition 3.1.([6, Theorem 1.1]) For $p \geqslant 7,0 \leqslant s<p-3$, the element $a_{3}^{s} h_{3,0} h_{2,1} h_{1,2} \in$ $E_{1}^{s+3, t, *}$ converges to the third Greek letter family element $\tilde{\gamma}_{s+3} \in E x t_{A}^{s+3, t}\left(\mathbb{Z}_{p}, \mathbb{Z}_{p}\right)$ in the May spectral sequence, where $t=(s+3) p^{2} q+(s+2) p q+(s+1) q+s$ and $\tilde{\gamma}_{s+3}$ converges to the $\gamma$ element $\gamma_{s+3} \in \pi_{(s+3) p^{2} q+(s+2) p q+(s+1) q-3} S$ in the Adams spectral sequence, where $\gamma_{s+3}=j j^{\prime} \bar{j} \gamma^{s+3} \bar{i} i^{\prime} i \in$ $\pi_{t-s-3} S$.

Proposition 3.2. Let $p \geqslant 7, n \geqslant 4,0 \leqslant s<p-5$, then in the May spectral sequence, $E_{1}^{s+6, p^{n} q+(s+3) p^{2} q+(s+4) p q+(s+1) q+s, *}=$ $\mathbb{Z}_{p}\left\{a_{3}^{s} h_{3,0} h_{2,1} h_{1,1} h_{1, n} b_{2,0}\right\}$.

Proof. First consider the structure of $E_{1}^{s+6, t^{\prime}, *}$ in the May spectral sequence, where $t^{\prime}=$ $p^{n} q+(s+3) p^{2} q+(s+4) p q+(s+1) q+s$. Consider $h=x_{1} x_{2} \cdots x_{m} \in E_{1}^{s+6, t^{\prime}, *}$, where $x_{i}$ is one of $a_{k}, h_{l, j}$ or $b_{u, z}, 0 \leqslant k \leqslant n+1,0 \leqslant l+j \leqslant n+1,0 \leqslant u+z \leqslant n, l>0, j \geqslant 0, u>0, z \geqslant 0$. Assume that deg $x_{i}=q\left(c_{i, n} p^{n}+c_{i, n-1} p^{n-1}+\cdots+c_{i, 0}\right)+e_{i}$, where $c_{i, j}=0$ or $1, e_{i}=1$ if $x_{i}=a_{k_{i}}$, 
or $e_{i}=0$. Then

$$
\left\{\begin{aligned}
\operatorname{deg} h & =\sum_{i=1}^{m} \operatorname{deg} x_{i} \\
& =q\left(\left(\sum_{i=1}^{m} c_{i, n}\right) p^{n}+\cdots+\left(\sum_{i=1}^{m} c_{i, 2}\right) p^{2}+\left(\sum_{i=1}^{m} c_{i, 1}\right) p+\left(\sum_{i=1}^{m} c_{i, 0}\right)\right)+\left(\sum_{i=1}^{m} e_{i}\right) \\
& =q\left(p^{n}+(s+3) p^{2}+(s+4) p+(s+1)\right)+s \\
\operatorname{dim} h & =\sum_{i=1}^{m} \operatorname{dim} x_{i}=s+6 .
\end{aligned}\right.
$$

By the facts that $\operatorname{dim} h_{i, j}=\operatorname{dim} a_{i}=1$ and $\operatorname{dim} b_{i, j}=2$, we know that $0<m \leqslant s+6$ from $\operatorname{dim} h=\sum_{i=1}^{m} \operatorname{dim} x_{i}=s+6$. Note that $c_{i, j}=0$ or $c_{i, j}=1, e_{i}=0$ or 1 and $m \leqslant s+6<p+1$. We have

$$
\left\{\begin{array}{l}
\sum_{i=1}^{m} e_{i}=s, \quad \sum_{i=1}^{m} c_{i, 0}=s+1, \quad \sum_{i=1}^{m} c_{i, 1}=s+4 \\
\sum_{i=1}^{m} c_{i, 2}=s+3, \quad\left(\sum_{i=1}^{m} c_{i, 3}\right) p^{3}+\cdots+\left(\sum_{i=1}^{m} c_{i, n}\right) p^{n}=p^{n}
\end{array}\right.
$$

Case 1. $0 \leqslant s<p-6$. Note that $m \leqslant s+6<p$. From (3.1) we have that $\sum_{i=1}^{m} e_{i}=s, \sum_{i=1}^{m} c_{i, 0}=$ $s+1, \sum_{i=1}^{m} c_{i, 1}=s+4, \sum_{i=1}^{m} c_{i, 2}=s+3, \sum_{i=1}^{m} c_{i, 3}=\cdots=\sum_{i=1}^{m} c_{i, n-1}=0, \sum_{i=1}^{m} c_{i, n}=1$. By (2.3), it is easy to see that there exists a factor $h_{i, n}$ or $b_{1, n-1}$ in $h$. By the graded commutativity of $E_{1}^{*, *, *}$, we can denote $h_{1, n}$ or $b_{1, n-1}$ by $x_{m}$. Then $h^{\prime}=x_{1} x_{2} \cdots x_{m-1} \in E_{1}^{l, t^{\prime}-p^{n} q, *}$, where $l=s+5$ or $s+4$ and we have

$$
\sum_{i=1}^{m-1} e_{i}=s, \sum_{i=1}^{m-1} c_{i, 0}=s+1, \sum_{i=1}^{m-1} c_{i, 1}=s+4, \sum_{i=1}^{m-1} c_{i, 2}=s+3 .
$$

We can get that $m \geqslant s+5$ from $\sum_{i=1}^{m-1} c_{i, 1}=s+4$. Meanwhile, we know that $m \leqslant s+6$, so $m=s+5$ or $s+6$. Since $\sum_{i=1}^{m-1} e_{i}=s, \operatorname{deg} h_{i, j} \equiv 0(\bmod q)(i>0, j \geqslant 0), \operatorname{deg} a_{i} \equiv 1(\bmod q)$ $(i \geqslant 0)$ and $\operatorname{deg} b_{i, j} \equiv 0(\bmod q)(i>0, j \geqslant 0)$, then by the graded commutativity of $E_{1}^{*, *, *}, h^{\prime}$ must have a factor $a_{j_{1}} a_{j_{2}} \cdots a_{j_{s}}\left(0 \leqslant j_{1} \leqslant j_{2} \leqslant \cdots \leqslant j_{s}\right)$. By the reason of the degrees of $a_{i}$ 's, we can suppose $h^{\prime}=a_{0}^{x} a_{1}^{y} a_{2}^{z} a_{3}^{k} x_{s+1} \cdots x_{m-1}$, where $0 \leqslant x, y, z, k \leqslant s, x+y+z+k=s$ and $m=s+5$ or $s+6$. From (3.2) we have

$$
y+z+k+\sum_{i=s+1}^{m-1} c_{i, 0}=s+1, z+k+\sum_{i=s+1}^{m-1} c_{i, 1}=s+4, k+\sum_{i=s+1}^{m-1} c_{i, 2}=s+3 .
$$

Subcase 1.1. If $h=x_{1} x_{2} \cdots x_{m-1} h_{1, n}$, then $h^{\prime}=x_{1} x_{2} \cdots x_{m-1} \in E_{1}^{s+5, t^{\prime}-p^{n} q, *}$.

When $m=s+5$, (3.3) can turn into that $\sum_{i=s+1}^{s+4} e_{i}=0, y+z+k+\sum_{i=s+1}^{s+4} c_{i, 0}=s+1, z+k+$ $\sum_{i=s+1}^{s+4} c_{i, 1}=s+4, k+\sum_{i=s+1}^{s+4} c_{i, 2}=s+3$. We can get that $k \geqslant s+3-\sum_{i=s+1}^{s+4} c_{i, 2} \geqslant s+3-4=s-1$ from $k+\sum_{i=s+1}^{s+4} c_{i, 2}=s+3$. Meanwhile, we have that $z+k \geqslant s$ from $z+k+\sum_{i=s+1}^{s+4} c_{i, 1}=s+4$. Note that $x+y+z+k=s$ and $0 \leqslant x, y, z, k \leqslant s$. Thus there are two possibilities that satisfy the two conditions. One is that $k=s, x=y=z=0$, the other is that $k=s-1, z=1, x=y=0$. If $k=s, x=y=z=0$, then $h^{\prime}=a_{3}^{s} \overline{x_{s+1} \cdots x_{s+4} \text { with } x_{s+1}} \cdots x_{s+4} \in E_{1}^{5,3 p^{2} q+4 p q+q, *}=\mathbb{Z}_{p}\left\{h_{3,0} h_{2,1} h_{1,1} b_{2,0}\right\}$.

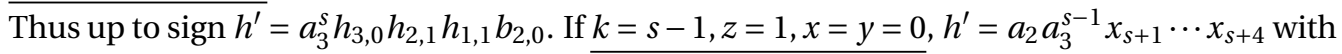
$x_{s+1} \cdots x_{s+4} \in E_{1}^{5,4 p^{2} q+4 p q+q, *}=0$. Thus the possibility $\underline{k=s-1, x}=1, y=z=0$ is impossible to exist.

When $m=s+6$, (3.3) can turn into that $\sum_{i=s+1}^{s+5} e_{i}=0, y+z+k+\sum_{i=s+1}^{s+5} c_{i, 0}=s+1, z+k+$ $\sum_{i=s+1}^{s+5} c_{i, 1}=s+4, k+\sum_{i=s+1}^{s+5} c_{i, 2}=s+3$. We can get that $k \geqslant s+3-\sum_{i=s+1}^{s+5} c_{i, 2} \geqslant s+3-5=s-2$ 
from $k+\sum_{i=s+1}^{s+5} c_{i, 2}=s+3$. Meanwhile, we can have that $z+k \geqslant s-1$ from $z+k+\sum_{i=s+1}^{s+5} c_{i, 1}=$ $s+4$. But we also know that $k \leqslant s$, so $s-2 \leqslant k \leqslant s$. There are seven possibilities satisfying the two conditions: $k \geqslant s-2$ and $z+k \geqslant s-1$. For the seven possibilities, we list a table as follows. (Let $t_{1}=(s+3-k) p^{2} q+(s+4-z-k) p q+(s+1-y-z-k) q$.)

\begin{tabular}{|c|c|c|c|c|c|c|}
\hline The possibility & $k$ & $z$ & $y$ & $x$ & $E_{1}^{5, t_{1}, *}$ & $\begin{array}{l}\text { The existence } \\
\text { of } x_{s+1} \cdots x_{s+5}\end{array}$ \\
\hline The 1st & $s-2$ & 1 & 1 & 0 & $E_{1}^{5, q\left(5 p^{2}+5 p+1\right), *}=0$ & Nonexistence \\
\hline The 2nd & $s-2$ & 1 & 0 & 1 & $E_{1}^{5, q\left(5 p^{2}+5 p+2\right), *}=0$ & Nonexistence \\
\hline The 3rd & $s-2$ & 2 & 0 & 0 & $E_{1}^{5, q\left(5 p^{2}+4 p+1\right), *}=0$ & Nonexistence \\
\hline The 4th & $s-1$ & 0 & 0 & 1 & $E_{1}^{5, q\left(4 p^{2}+5 p+2\right), *}=0$ & Nonexistence \\
\hline The 5th & $s-1$ & 0 & 1 & 0 & $E_{1}^{5, q\left(4 p^{2}+5 p+1\right), *}=0$ & Nonexistence \\
\hline The 6th & $s-1$ & 1 & 0 & 0 & $E_{1}^{5, q\left(4 p^{2}+4 p+1\right), *}=0$ & Nonexistence \\
\hline The 7th & $\mathrm{s}$ & 0 & 0 & 0 & $E_{1}^{5, q\left(3 p^{2}+4 p+1\right), *}$ & Nonexistence \\
\hline
\end{tabular}

From the above table, it follows that when $m=s+6, h^{\prime}$ can not exist.

From the above argument, we get that $h$ exists and up to sign $h=a_{3}^{s} h_{3,0} h_{2,1} h_{1,1} b_{2,0} h_{1, n}$.

Subcase 1.2. If $h=x_{1} x_{2} \cdots x_{m-1} b_{1, n-1}$, then $h^{\prime}=x_{1} x_{2} \cdots x_{m-1} \in E_{1}^{s+4, t^{\prime}-p^{n} q, *}$.

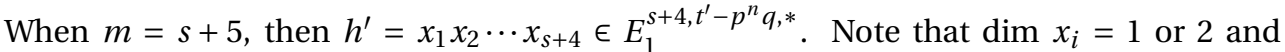
$\operatorname{dim} h^{\prime}=s+4$. It is easy to see that $h^{\prime}=x_{1} x_{2} \cdots x_{s+4} \in E\left(h_{m, i} \mid m>0, i \geqslant 0\right) \otimes P\left(a_{n} \mid n \geqslant 0\right)$. From (3.3), we have that $z+k \geqslant s$ and $k \geqslant s-1$. we can get that there are two possibilities satisfying the two conditions. One is that $k=s, x=y=z=0$, the other is that $k=s-1, z=1, x=y=0$. If $k=s, x=y=z=0, h^{\prime}=a_{3}^{s} x_{s+1} \cdots x_{s+4}$ with $x_{s+1} \cdots x_{s+4} \in E_{1}^{4,3 p^{2} q+4 p \overline{q+q, *}=0 . \text { If } k=s-1, z=1, x=y=0,}$ then $h^{\prime}=a_{2} a_{3}^{s-1} x_{s+1} \cdots x_{s+4}$ with $x_{s+1} \cdots x_{s+4} \in E_{1}^{4,4 p^{2} q+4 p q+q, *}=0$. Thus in this case $h^{\prime}$ is impossible to exist either.

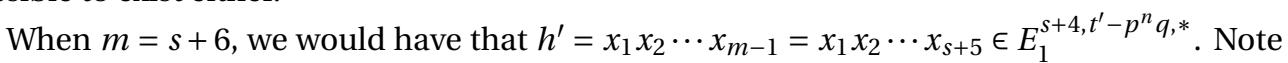
that $\operatorname{dim} x_{i}=1$ or 2 . It is easy to see that $m$ is impossible to equal $s+6$.

From the above argument, we get that $h^{\prime}$ is impossible to exist. Then it follows that $h=$ $x_{1} x_{2} \cdots x_{m-1} b_{1, n-1}$ is impossible to exist.

From Subcases 1.1 and 1.2, we see that when $0 \leqslant s<p-6, h$ exists such that up to sign $h=a_{3}^{s} h_{3,0} h_{2,1} h_{1,1} h_{1, n} b_{2,0}$, i.e., $E_{1}^{s+6, t^{\prime}, *}=\mathbb{Z}_{p}\left\{a_{3}^{s} h_{3,0} h_{2,1} h_{1,1} h_{1, n} b_{2,0}\right\}$.

Case 2. $s=p-6$. Then $m \leqslant s+6=p-6+6=p$. From (3.1) we have $\left(\sum_{i=1}^{m} c_{i, 3}\right)+\left(\sum_{i=1}^{m} c_{i, 4}\right) p+$ $\cdots+\left(\sum_{i=1}^{m} c_{i, n}\right) p^{n-3}=p^{n-3}$. Therefore, $p \mid \sum_{i=1}^{m} c_{i, 3}$. Note that $c_{i, 3}=0$ or $1, m \leqslant p$, it is easy to know that $\sum_{i=1}^{m} c_{i, 3}=0$ or $p$.

Subcase 2.1. $\sum_{i=1}^{m} c_{i, 3}=0$. When $n=4$, it is easy to get that $\sum_{i=1}^{m} c_{i, 4}=1$, so there exists a factor $h_{1, n}$ or $b_{1, n-1}$ in $h$. 
When $n>4$, then $\left(\sum_{i=1}^{m} c_{i, 4}\right) p^{4}+\cdots+\left(\sum_{i=1}^{m} c_{i, n}\right) p^{n}=p^{n}$, so $\left(\sum_{i=1}^{m} c_{i, 4}\right)+\left(\sum_{i=1}^{m} c_{i, 5}\right) p+\cdots+$ $\left(\sum_{i=1}^{m} c_{i, n}\right) p^{n-4}=p^{n-4}$. Similarly we know that $\sum_{i=1}^{m} c_{i, 4}=0$ or $=p$. We claim that if $\sum_{i=1}^{m} c_{i, 3}=$ 0 , then $\sum_{i=1}^{m} c_{i, 4}=0$. For otherwise, we would have $\sum_{i=1}^{m} c_{i, 4}=p$, then $m=p$. For each $1 \leqslant$ $i \leqslant m, \operatorname{deg} x_{i}=$ higher terms $+p^{4} q+$ lower terms. Since $\sum_{i=1}^{p} e_{i}=p-6, \operatorname{deg} b_{i, j} \equiv 0(\bmod q)$ $(i>0, j \geqslant 0), \operatorname{deg} a_{i} \equiv 1(\bmod q)(i \geqslant 0)$ and $\operatorname{deg} h_{i, j} \equiv 0(\bmod q)(i>0, j \geqslant 0)$, then by the graded commutativity of $E_{1}^{*, *, *}$, there would exist a factor $a_{j_{1}} a_{j_{2}} \cdots a_{j_{p-6}}\left(0 \leqslant j_{1} \leqslant j_{2} \leqslant \cdots \leqslant\right.$ $\left.j_{p-6} \leqslant n+1\right)$ among $x_{i}$ 's such that for any $1 \leqslant i \leqslant p-6, j_{i} \geqslant 5$ and deg $a_{j_{i}}=$ higher terms $+p^{4} q+p^{3} q+p^{2} q+p q+q+1$. It is obvious that $\sum_{i=1}^{m} c_{i, 3} \geqslant p-6$ which contradicts to $\sum_{i=1}^{m} c_{i, 3}=$ 0 , thus the claim is proved. By induction on $j$ we can get $\sum_{i=1}^{m} c_{i, j}=0(4 \leqslant j \leqslant n-1)$, so $\sum_{i=1}^{m} c_{i, n}=1$. By (2.3), it follows that there is a factor $h_{1, n}$ or $b_{1, n-1}$ in $h$.

In all, for $n \geqslant 4$, there is a factor $h_{1, n}$ or $b_{1, n-1}$ in $h$. By the graded commutativity of $E_{1}^{*, *, *}$, we can denote $h_{1, n}$ or $b_{1, n-1}$ by $x_{m}$. By an argument similar to that used in the proof in Case 1 , we can show that $h$ exists such that $h=a_{3}^{p-6} h_{3,0} h_{2,1} h_{1,1} h_{1, n} b_{2,0}$ up to sign.

Subcase 2.2. $\sum_{i=1}^{m} c_{i, 3}=p$. By $m \leqslant s+6=p$ and $c_{i, 3}=0$ or 1 , we have that $m=p$. By $\operatorname{dim} x_{i}=1$ or 2 , we have that $\operatorname{dim} x_{i}=1$ from $\operatorname{dim} h=\sum_{i=1}^{p} \operatorname{dim} x_{i}=p$. Thus $h \in E\left(h_{m, i} \mid m>\right.$ $0, i \geqslant 0) \otimes P\left(a_{n} \mid n \geqslant 0\right)$. We claim that $\sum_{i=1}^{p} c_{i, 3}=p$ is impossible to exist. For otherwise, we would have that for each $1 \leqslant i \leqslant p$, deg $x_{i}=$ higher terms $+p^{3} q+$ lower terms. At the same time, from $\sum_{i=1}^{p} c_{i, 1}=p-2$, we know that there would be $p-2 x_{i}$ 's in $h$ with deg $x_{i}=$ higher terms $+p^{1} q+$ lower terms. Note that $\operatorname{deg} h_{s, i}=\left(p^{s+i-1}+\cdots+p^{i}\right) q(s>0, i \geqslant 0)$ and $\operatorname{deg} a_{i}=$ $\left(p^{i-1}+\cdots+1\right) q+1(i>0)$. Thus there would be $p-2 x_{i}$ 's with deg $x_{i}=$ higher terms $+p^{3} q+$ $p^{2} q+p^{1} q+$ lower terms. And it would follow that $\sum_{i=1}^{p} c_{i, 2} \geqslant p-2$ which would contradict to $\sum_{i=1}^{p} c_{i, 2}=p-3$. The claim is proved.

From Subcases 2.1 and 2.2, we get when $s=p-6$,

$$
E_{1}^{p, t^{\prime}, *}=\mathbb{Z}_{p}\left\{a_{3}^{p-6} h_{3,0} h_{2,1} h_{1,1} h_{1, n} b_{2,0}\right\}
$$

From Cases 1 and 2, the proposition follows.

Proposition 3.3. Let $p \geqslant 7, n \geqslant 4,0 \leqslant s<p-5$, then the product

$$
b_{0} h_{1} h_{n} \tilde{\gamma}_{s+3} \neq 0 \in E x t_{A}^{s+7, p^{n} q+(s+3) p^{2} q+(s+4) p q+(s+1) q+s}\left(\mathbb{Z}_{p}, \mathbb{Z}_{p}\right) .
$$

Proof. It is known that $h_{1, n}, b_{1, n}$ and $a_{3}^{s} h_{3,0} h_{2,1} h_{1,2} \in E_{1}^{*, *, *}$ are permanent cycles in the May spectral sequence and converge nontrivially to $h_{n}, b_{n}, \tilde{\gamma}_{s+3} \in E x t_{A}^{*, *}\left(\mathbb{Z}_{p}, \mathbb{Z}_{p}\right)$ for $n \geqslant 0$ respectively (see Proposition 3.1).

By (2.2), we have

$$
\begin{aligned}
d_{1}( & \left.a_{3}^{s} h_{3,0} h_{2,1} h_{1,1} h_{1, n} b_{2,0}\right) \\
& =d_{1}\left(a_{3}^{s} h_{3,0} h_{2,1}\right) h_{1,1} h_{1, n} b_{2,0}+(-1)^{s+2}\left(a_{3}^{s} h_{3,0} h_{2,1}\right) d_{1}\left(h_{1,1} h_{1, n} b_{2,0}\right) \\
& =d_{1}\left(a_{3}^{s} h_{3,0} h_{2,1}\right) h_{1,1} h_{1, n} b_{2,0}+0 \\
& =\left[d_{1}\left(a_{3}^{s}\right) h_{3,0} h_{2,1}+(-1)^{s+1} h_{2,0} h_{1,2} h_{2,1}\right] h_{1,1} h_{1, n} b_{2,0} \\
& =d_{1}\left(a_{3}^{s}\right) h_{3,0} h_{2,1} h_{1,1} h_{1, n} b_{2,0}+(-1)^{s+1} h_{2,0} h_{1,2} h_{2,1} h_{1,1} h_{1, n} b_{2,0} .
\end{aligned}
$$


By induction on $s$, we can have

$$
d_{1}\left(a_{3}^{s}\right) h_{3,0} h_{2,1} h_{1,1} h_{1, n} b_{2,0}=(-1)^{s} s a_{3}^{s-1} a_{2} h_{1,2} h_{3,0} h_{2,1} h_{1,1} h_{1, n} b_{2,0}
$$

Thus we have that

$$
\begin{aligned}
& d_{1}\left(a_{3}^{s} h_{3,0} h_{2,1} h_{1,1} h_{1, n} b_{2,0}\right) \\
& \quad=(-1)^{s} s a_{3}^{s-1} a_{2} h_{1,2} h_{3,0} h_{2,1} h_{1,1} h_{1, n} b_{2,0}+(-1)^{s+1} h_{2,0} h_{1,2} h_{2,1} h_{1,1} h_{1, n} b_{2,0} \neq 0
\end{aligned}
$$

and $d_{1}\left(a_{3}^{s} h_{3,0} h_{2,1} h_{1,1} h_{1, n} b_{2,0}\right) \neq b_{1,0} h_{1,0} h_{1, n} a_{3}^{s} h_{3,0} h_{2,1} h_{1,2}$ up to nonzero scalar. Thus we have that $E_{r}^{s+6, t^{\prime}, *}=0$ for all $r \geqslant 2$. It follows that $b_{1,0} h_{1,1} h_{1, n} a_{3}^{s} h_{3,0} h_{2,1} h_{1,2} \in E_{r}^{s+7, t^{\prime}, *}$ does not bound. Then the product $b_{1,0} h_{1,1} h_{1, n} a_{3}^{s} h_{3,0} h_{2,1} h_{1,2} \in E_{r}^{s+7, t^{\prime}, *}$ is a permanent cycle in the May spectral sequence and converges nontrivially to $b_{0} h_{1} h_{n} \tilde{\gamma}_{s+3} \in E x t_{A}^{s+7, t^{\prime}}\left(\mathbb{Z}_{p}, \mathbb{Z}_{p}\right)$. It follows that $b_{0} h_{1} h_{n} \tilde{\gamma}_{s+3} \neq 0 \in E x t_{A}^{s+7, t^{\prime}}\left(\mathbb{Z}_{p}, \mathbb{Z}_{p}\right)$.

0 .

Proposition 3.4. Let $p \geqslant 7, n \geqslant 4,0 \leqslant s<p-5,2 \leqslant r \leqslant s+7$, then $\operatorname{Ext}_{A}^{s+7-r, q\left(p^{n}+(s+3) p^{2}+(s+4) p+(s+1)\right)+(s-r+1)}\left(\mathbb{Z}_{p}, \mathbb{Z}_{p}\right)=$

Proof. The proof is divided into two cases.

Case 1. $r=s+7$ or $s+6$. By [4], it is easy to get that in these cases

$$
E x t_{A}^{s+7-r, q\left(p^{n}+(s+3) p^{2}+(s+4) p+(s+1)\right)+(s-r+1)}\left(\mathbb{Z}_{p}, \mathbb{Z}_{p}\right)=0
$$

Case 2. $2 \leqslant r<s+6$. To prove $E x t_{A}^{s+7-r, t^{\prime \prime}}\left(\mathbb{Z}_{p}, \mathbb{Z}_{p}\right)=0$, it suffices to prove that in the May spectral sequence $E_{1}^{s+7-r, t^{\prime \prime}, *}=0$, where $t^{\prime \prime}=q\left(p^{n}+(s+3) p^{2}+(s+4) p+(s+1)\right)+(s-r+1)$. Consider $h=x_{1} x_{2} \cdots x_{m} \in E_{1}^{s+7-r, t^{\prime \prime}, *}$, where $x_{i}$ is one of $a_{k}, h_{l, j}$ or $b_{u, z}, 0 \leqslant k \leqslant n+1,0 \leqslant$ $l+j \leqslant n+1,0 \leqslant u+z \leqslant n, l>0, j \geqslant 0, u>0, z \geqslant 0$. Assume that deg $x_{i}=q\left(c_{i, n} p^{n}+c_{i, n-1} p^{n-1}+\right.$ $\left.\cdots+c_{i, 0}\right)+e_{i}$, where $c_{i, j}=0$ or $1, e_{i}=1$ if $x_{i}=a_{k_{i}}$, or $e_{i}=0$. Then

$$
\left\{\begin{aligned}
\operatorname{deg} h & =\sum_{i=1}^{m} \operatorname{deg} x_{i} \\
& =q\left(\left(\sum_{i=1}^{m} c_{i, n}\right) p^{n}+\cdots+\left(\sum_{i=1}^{m} c_{i, 2}\right) p^{2}+\left(\sum_{i=1}^{m} c_{i, 1}\right) p+\left(\sum_{i=1}^{m} c_{i, 0}\right)\right)+\left(\sum_{i=1}^{m} e_{i}\right) \\
& =q\left(p^{n}+(s+3) p^{2}+(s+4) p+(s+1)\right)+(s-r+1), \\
\operatorname{dim} h & =\sum_{i=1}^{m} \operatorname{dim} x_{i}=s+7-r .
\end{aligned}\right.
$$

By $\operatorname{dim} x_{i}=1$ or 2 and $2 \leqslant r<s+6$, we can get that $m \leqslant s+7-r \leqslant s+7-2=s+5<p$ from $\operatorname{dim} h=\sum_{i=1}^{m} \operatorname{dim} x_{i}=s+7-r$. We claim that $s-r+1 \geqslant 0$. Otherwise we would have $p>\sum_{i=1}^{m} e_{i}=q+(s+1-r) \geqslant q-5 \geqslant p$ by $p \geqslant 7$. That is impossible. The claim follows. By $c_{i, j}=0$ or $1, e_{i}=0$ or 1 and $m<p$, we have that $\sum_{i=1}^{m} e_{i}=s-r+1, \sum_{i=1}^{m} c_{i, 0}=s+1, \sum_{i=1}^{m} c_{i, 1}=$ $s+4, \sum_{i=1}^{m} c_{i, 2}=s+3, \sum_{i=1}^{m} c_{i, 3}=\cdots=\sum_{i=1}^{m} c_{i, n-1}=0, \sum_{i=1}^{m} c_{i, n}=1$. It is easy to see that there exists a $h_{1, n}$ or $b_{1, n-1}$ in $h$. We denote $h_{1, n}$ or $b_{1, n-1}$ by $x_{m}$, then $h^{\prime}=x_{1} \cdots x_{m-1} \in E_{1}^{l, t^{\prime \prime}-p^{n} q \text {,*, }}$ where $l=s+6-r$ or $s+5-r$. And we have that $\sum_{i=1}^{m-1} e_{i}=s-r+1, \sum_{i=1}^{m-1} c_{i, 0}=s+1, \sum_{i=1}^{m-1} c_{i, 1}=$ $s+4, \sum_{i=1}^{m-1} c_{i, 2}=s+3$. 
Subcase 2.1. If $h=x_{1} x_{2} \cdots x_{m-1} h_{1, n}, h^{\prime}=x_{1} x_{2} \cdots x_{m-1} \in E_{1}^{s+6-r, t^{\prime \prime}-p^{n} q, *}$. When $r \geqslant 3$, from $s+6-r \leqslant s+3<\sum_{i=1}^{m-1} c_{i, 1}=s+4$ we can get that $E_{1}^{s+6-r, t^{\prime \prime}-p^{n} q, *}=0$ by Proposition 2.1. When $r=2$, we can easily show that $E_{1}^{s+4, t^{\prime \prime}-p^{n} q, *}=0$ by an argument similar to that used in Case 1 of the proof of Proposition 3.2.

Subcase 2.2. If $h=x_{1} x_{2} \cdots x_{m-1} b_{1, n-1}, h^{\prime}=x_{1} x_{2} \cdots x_{m-1} \in E_{1}^{s+5-r, t^{\prime \prime}-p^{n} q, *}$. Note that $2 \leqslant$ $r<s+7$. From $s+5-r \leqslant s+3<\sum_{i=1}^{m-1} c_{i, 1}=s+4$, we have that $E_{1}^{s+5-r, t^{\prime \prime}-p^{n} q, *}=0$ by Proposition 2.1 .

From Cases 1 and 2, we have that $E x t_{A}^{s+7-r, t^{\prime \prime}}\left(\mathbb{Z}_{p}, \mathbb{Z}_{p}\right)=0$.

Proof of Theorem 1.4. From [2], $\left(b_{0} h_{n}+h_{1} b_{n-1}\right) \in E x t_{A}^{3, p^{n} q+p q}\left(\mathbb{Z}_{p}, \mathbb{Z}_{p}\right)$ is a permanent cycle in the Adams spectral sequence and converges to a nontrivial element $j \xi_{n} \in \pi_{p^{n} q+p q-3} S$ for $n \geqslant 3$. Let $\gamma: \Sigma^{q\left(p^{2}+p+1\right)} V(2) \rightarrow V(2)$ be the $v_{3}$-map and consider the following composition of maps

$$
\bar{f}: \Sigma^{p^{n} q+2 p q-1} S \stackrel{j \xi_{n}}{\longrightarrow} \Sigma^{p q-1} S \stackrel{j^{\prime} \beta i^{\prime} i}{\longrightarrow} M^{j j^{\prime} \dot{j} \stackrel{\gamma}{s+3}^{\longrightarrow} i^{\prime}} \Sigma^{-(s+3) p^{2} q-(s+2) p q-(s+1) q+3} S .
$$

It is known that $i_{*}\left(h_{1}\right) \in E x t_{A}^{1, p q}\left(H^{*} M, \mathbb{Z}_{p}\right)$ converges nontrivially to the map $g=j^{\prime} \beta i^{\prime} i \in$ $\left[\Sigma^{p q-1} S, M\right]$ in the Adams spectral sequence. Meanwhile, up to nonzero scaler $j \xi_{n}$ is represented by $\left(b_{0} h_{n}+h_{1} b_{n-1}\right) \in E x t_{A}^{* * *}\left(\mathbb{Z}_{p}, \mathbb{Z}_{p}\right)$ in the Adams spectral sequence. Then the above $\bar{f}$ is represented up to nonzero scalar by $\left.\bar{c}=\left(j j^{\prime} \bar{j} \gamma_{*}\right)^{s+3} \bar{i} i^{\prime}\right)_{*} i_{*}\left(h_{1}\left(b_{0} h_{n}+h_{1} b_{n-1}\right)\right)=\left(j j^{\prime} \bar{j} \gamma^{s+3} \bar{i} i^{\prime} i\right)_{*}\left(b_{0} h_{1} h_{n}\right)$.

From Proposition 3.1 and the knowledge of Yoneda products we know that the composition $\operatorname{Ext}_{A}^{0, *}\left(\mathbb{Z}_{p}, \mathbb{Z}_{p}\right) \stackrel{\left(j j^{\prime} \bar{j}\right)_{*}}{\stackrel{\left(\gamma_{*}\right)^{s+3}\left(\bar{i} i^{\prime} i\right)_{*}}{\longrightarrow}} \operatorname{Ext}_{A}^{s+3, *+(s+3) p^{2} q+(s+2) p q+(s+1) q+s}\left(\mathbb{Z}_{p}, \mathbb{Z}_{p}\right)$ is a multiplication up to nonzero scalar by $\tilde{\gamma}_{s+3} \in \operatorname{Ext}_{A}^{s+3,(s+3) p^{2} q+(s+2) p q+(s+1) q+s}\left(\mathbb{Z}_{p}, \mathbb{Z}_{p}\right)$. Hence, $\bar{f}$ is represented by $\bar{c}=\tilde{\gamma}_{s+3} b_{0} h_{1} h_{n} \neq 0 \in \operatorname{Ext}_{A}^{s+7, *}\left(\mathbb{Z}_{p}, \mathbb{Z}_{p}\right)$ up to nonzero scalar in the Adams spectral sequence (see Proposition 3.3). Moreover, from Proposition 3.4, we can see that $b_{0} h_{1} h_{n} \tilde{\gamma}_{s+3}$ cannot be hit by any differential in the Adams spectral sequence and so the corresponding homotopy element $\bar{f} \in \pi_{*} S$ is nontrivial and of order $p$. This finishes the proof of Theorem 1.4 .

\section{References}

[1] J. K. Lin and Q. B. Zheng, A new family of filtration seven in the stable homotopy of spheres, Hiroshima Math. J. 28(1998), 183-205.

[2] J. K. Lin, New families in the stable homotopy of spheres revisited, Acta Math. Sin. (Engl. Ser.) 18(2002), 95-106.

[3] J. K. Lin, A new family of filtration three in the stable homotopy of spheres, Hiroshima Math. J. 31(2001), 477-492.

[4] A. Liulevicius, The factorizations of cyclic reduced powers by secondary cohomology operations, Memo. Amer. Math. Soc. 42(1962), 112pp.

[5] D. C. Ravenel, Complex Cobordism and Stable Homotopy Groups of Spheres, Academic Press, Orlando, 1986. 
[6] X. G. Liu, A nontrivial product in the stable homotopy groups of spheres, Sci. China Ser. A 47(2004), 831-841.

[7] H. R. Miller, D. C. Ravenel, and W. S. Wilson, Periodic phenomena in the Adams-Novikov spectral sequence, Ann. Math. (2) 106(1977), 469-516.

Department of Mathematics, Nankai University, Tianjin 300071, P.R. China.

E-mail:xgliu@nankai.edu.cn

Department of Mathematics, Tianjin University, Tianjin 300072, P.R. China.

E-mail: jinyinglong@eyou.com 